\title{
Model-based Analysis of the Effects of Thioridazine Enantiomers on the Rabbit Papillary Action Potential
}

\author{
Ask S Jensen, Cristian P Pennisi, Cristian Sevcencu, Jørn B Christensen, Jette E Kristiansen, \\ Johannes J Struijk \\ Aalborg University, Aalborg, Denmark
}

\begin{abstract}
Aims: We investigated mechanisms underlying the effects of the thioridazine enantiomers on the rabbit papillary action potential duration (AP, APD).

Methods: An adapted computational model of the rabbit ventricular action potential was used to carry out a model-based analysis of transmembrane AP recordings from isolated right ventricular papillary muscles from 21 rabbits in four groups: control, (-)-thioridazine, (+)thioridazine, and racemate. Drug effects were determined using an inverse method and a forward method. Effects were modeled by inhibition of the $\mathrm{I}_{\mathrm{Kr}}$ and $\mathrm{I}_{\mathrm{CaL}}$ currents.

Results: Simultaneous inhibition of $\mathrm{I}_{\mathrm{Kr}}$ and $\mathrm{I}_{\mathrm{CaL}}$ resulted in a more accurate description of the observed drug effects than could $\mathrm{I}_{\mathrm{Kr}}$ inhibition alone. The following values of $\mathrm{I}_{\mathrm{Kr}}$ inhibition at $10 \mathrm{mg} \mathrm{L}^{-1}$ were determined. Forward method: Racemate $=45 \%,(-)$-thioridazine $=0 \%$, and $(+)$-thioridazine $=85 \%$. Inverse method: (-)thioridazine $=35 \%,(+)$-thioridazine $=80 \%$.

Conclusion: $\mathrm{I}_{\mathrm{Kr}}$ inhibition accurately described the observed APD prolongation, and the identified levels of $\mathrm{I}_{\mathrm{Kr}}$ inhibition were plausible when compared to literature. Both methods found (-)-thioridazine to cause less $\mathrm{I}_{\mathrm{Kr}}$ inhibition than $(+)$-thioridazine or the racemate. These results indicate that the prolonging effects observed in the experiment may be related to $\mathrm{I}_{\mathrm{Kr}}$ inhibition.
\end{abstract}

\section{Introduction}

Investigation into the effects of the thioridazine (+)and (-)-enantiomers on the isolated rabbit papillary APD showed that $(+)$-thioridazine and the racemate cause greater APD prolongation than (-)-thioridazine [1]. In order to further analyze possible mechanisms underlying the effects of these drugs, a mathematical model of the rabbit ventricular AP [2] was adapted to reproduce baseline APs [3]. Based on available evidence and a characterization of the sensitivity of the APD to changes in major transmembrane current conductances [3], it is possible that the observed effects may be related to simultaneous inhibition of the rapid component of the delayed rectifier potassium current $\left(\mathrm{I}_{\mathrm{Kr}}\right)$ and $\mathrm{L}$ type calcium current $\left(\mathrm{I}_{\mathrm{CaL}}\right)$.

Using the baseline adapted model of the rabbit ventricular AP, the aim of this study was to investigate the ability of the model to explain the measured drug effects as well as to determine levels of $I_{\mathrm{Kr}}$ inhibition due to each drug.

\section{Methods and materials}

Data collection was previously described in detail [1]. APs were recorded in right ventricular papillary muscles isolated from 21 female New Zealand white rabbits. Drug effects were investigated at $0,0.1,1$, and $10 \mathrm{mg} \mathrm{L}^{-1}$ at 2.0 and $0.5 \mathrm{~Hz}$ pacing. Rabbits were divided into four groups: control $(\mathrm{N}=5)$, racemate $(\mathrm{N}=6),(+)$-thioridazine $(\mathrm{N}=4)$, and (-)-thioridazine $(\mathrm{N}=6)$. The drug effect in comparison with control $(\Delta \Delta$-APD) was calculated. The observed effects are shown in Figure 1 and Figure 2. For the racemate, a dose-dependent APD prolongation was observed at both pacing rates. For (-)-thioridazine, a minor shortening was observed at $0.5 \mathrm{~Hz}$ pacing at 0.1 $\mathrm{mg} \mathrm{L}^{-1}$ independent of dose. At $2 \mathrm{~Hz}$ pacing, no change was observed. For (+)-thioridazine, shortening was observed at $0.1 \mathrm{mg} \mathrm{L}^{-1}$ dose which was greater at $0.5 \mathrm{~Hz}$ than $2 \mathrm{~Hz}$, and dose dependent prolongation was observed from 0.1 to $10 \mathrm{mg} \mathrm{L}^{-1}$. Thus, it appears that drug-induced APD change was primarily caused by at least two separate effects: a dose-dependent APD prolongation at both 2.0 and $0.5 \mathrm{~Hz}$ pacing, and a shortening that did not display clear dose-dependence within the investigated concentration range. The shortening effect was also stronger at the slower pacing rate.

The conductance of the $\mathrm{I}_{\mathrm{CaL}}$ and $\mathrm{I}_{\mathrm{Kr}}$ channels were calculated to reproduce $\Delta \Delta$-APD effects. The fit error was quantified as the RMSE between modeled and measured $\mathrm{APD}_{30}, \mathrm{APD}_{60}$, and $\mathrm{APD}_{90}$ change at both 2 and $0.5 \mathrm{~Hz}$ pacing. $\mathrm{I}_{\mathrm{CaL}}$ and $\mathrm{I}_{\mathrm{Kr}}$ inhibition has opposing effects on APD. For this reason, several methods were used to select or keep constant one of these inhibitions, while the inhibition for the other channel was determined by optimization. Overall, this was accomplished through two 

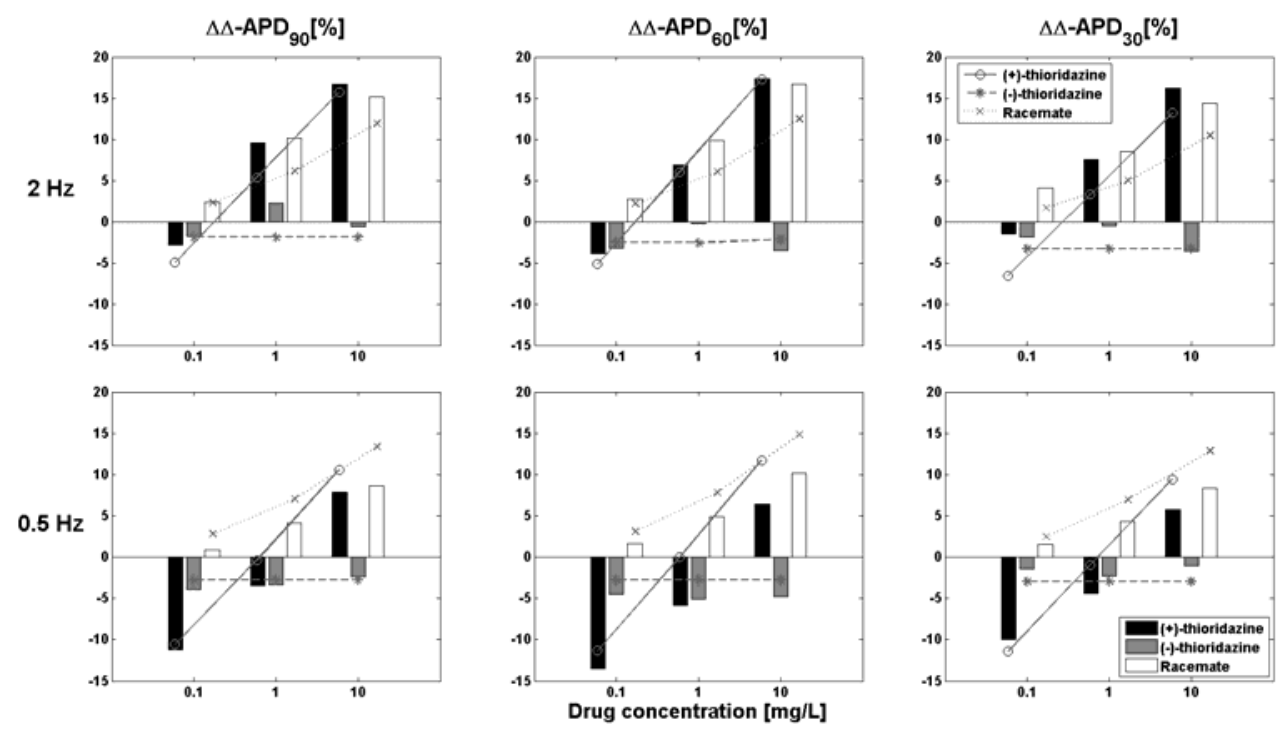

Figure 1: forward method results. Bars indicate mean measured $\Delta \Delta$-APD, lines indicate model values.
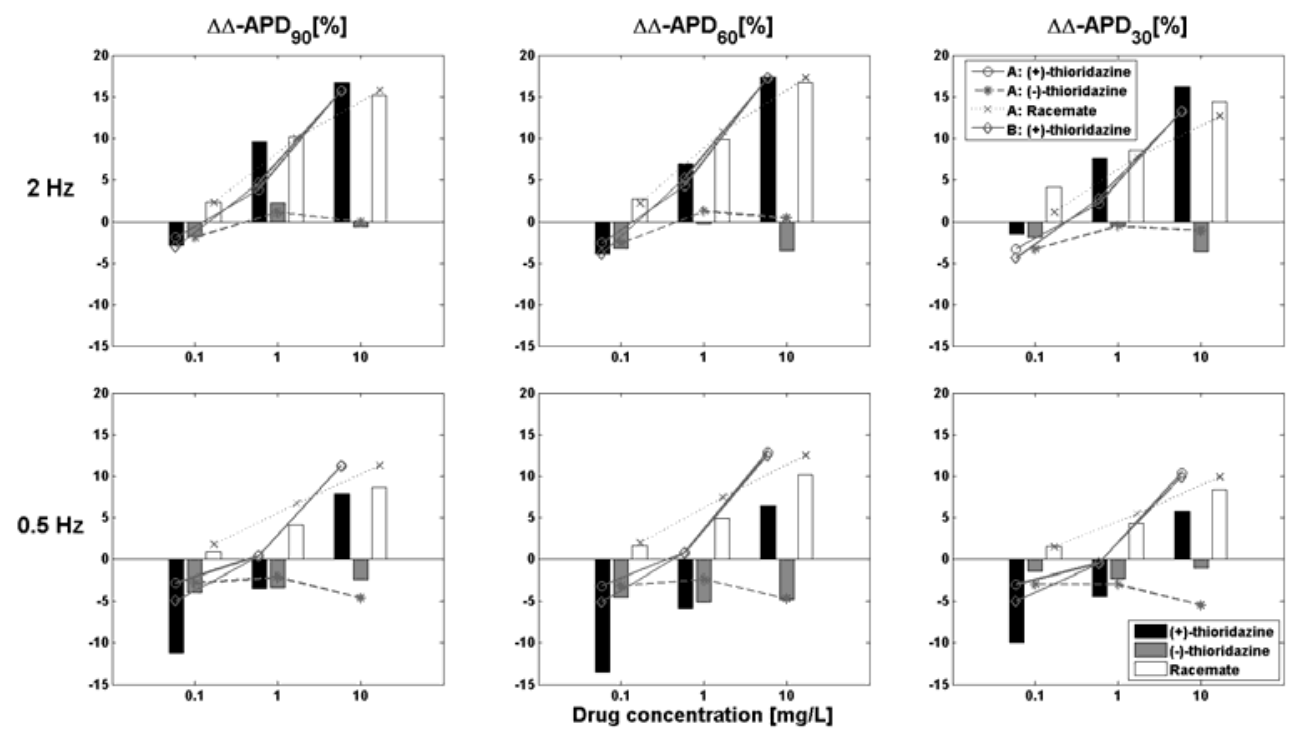

Figure 2: inverse method results. Bars indicate mean measured $\Delta \Delta-\mathrm{APD}$, lines indicate model/literature values.

approaches based on different assumptions. These will be referred to as the forward- and the inverse methods.

\subsection{Forward method}

The forward method assumed small $\mathrm{I}_{\mathrm{Kr}}$ inhibition at $0.1 \mathrm{mg} \mathrm{\textrm {L } ^ { - 1 }}$. This agreed well with experimental observations. It also assumed saturated $\mathrm{I}_{\mathrm{CaL}}$ inhibition at $0.1 \mathrm{mg} \mathrm{L}^{-1}$ as no dose-dependent shortening was observed with increasing dose. First the $\mathrm{I}_{\mathrm{CaL}}$ inhibition at $0.1 \mathrm{mg} \mathrm{L}^{-1}$ was determined by optimization. This level of $\mathrm{I}_{\mathrm{CaL}}$ inhibition was set to persist, and the $\mathrm{I}_{\mathrm{Kr}}$ inhibition for each drug concentration was determined by optimization. As the dose dependent prolongation caused by $(+)-$ thioridazine was stronger than that caused by the racemate, and as APD appeared to be affected by two opposing effects, we assumed that (+)-thioridazine caused $\mathrm{I}_{\mathrm{Kr}}$ inhibition slightly greater than the racemate at $0.1 \mathrm{mg}$ $\mathrm{L}^{-1}$, and that the effect of this $\mathrm{I}_{\mathrm{Kr}}$ inhibition was masked by the opposing APD shortening due to $\mathrm{I}_{\mathrm{CaL}}$ inhibition. The $\mathrm{I}_{\mathrm{Kr}}$ inhibition due to (+)-thioridazine at higher concentrations was determined by optimization.

\subsection{Inverse method}

The overall strategy for the inverse method was to 

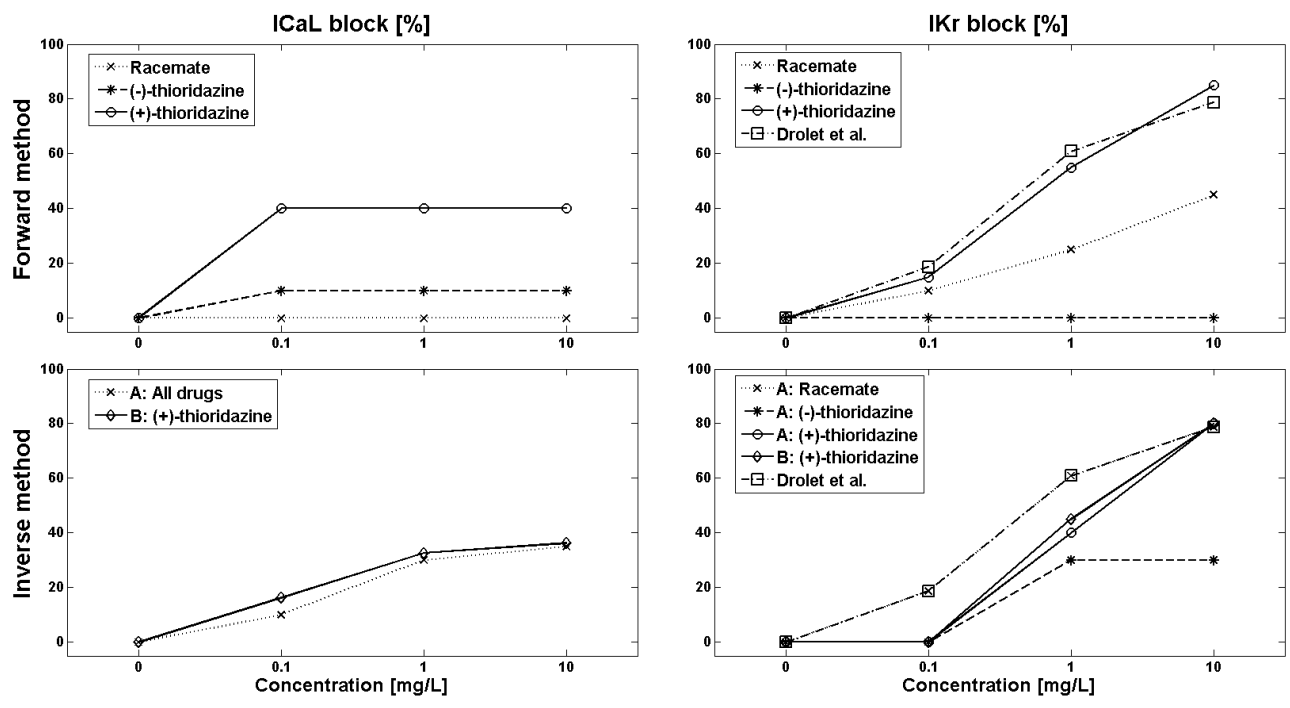

Figure 3: inhibition of $\mathrm{I}_{\mathrm{CaL}}$ and $\mathrm{I}_{\mathrm{Kr}}$ for each method. $\mathrm{I}_{\mathrm{Kr}}$ inhibition is compared to values from the literature.

determine levels of $\mathrm{I}_{\mathrm{CaL}}$ inhibition for the racemate based on values of $\mathrm{I}_{\mathrm{Kr}}$ and $\mathrm{I}_{\mathrm{Ks}}$ inhibition available in the literature. Two different versions of the inverse method were applied; A and B. These are described below:

A: Values of racemate induced $\mathrm{I}_{\mathrm{Kr}}$ and $\mathrm{I}_{\mathrm{Ks}}$ inhibition were obtained from dose-response curves of inhibition [4]. These were derived from patch clamp experiments in isolated guinea pig ventricular myocytes and agreed well the with concentration dependence of APD prolongation in our data. Given these levels of potassium current inhibition, $\mathrm{I}_{\mathrm{CaL}}$ inhibition was determined for the racemate using our experimental data. A dose-response curve was fitted to the determined values of $\mathrm{I}_{\mathrm{Ca}}$ inhibition using logistic regression with a standard dose-response curve (Hill coefficient $n=1$ ). Assuming that the enantiomers cause identical $\mathrm{I}_{\mathrm{Ca}}$ inhibition, the same levels of inhibition were set for each. The $\mathrm{I}_{\mathrm{Kr}}$ inhibition was then determined by optimization.

B: Greater APD shortening was observed for (+)thioridazine than for (-)-thioridazine. In order to examine if difference in $\mathrm{I}_{\mathrm{CaL}}$ inhibiting effect between the enantiomers could explain this observation, an additional variation of the inverse method was used for $(+)$ thioridazine. $\mathrm{I}_{\mathrm{Kr}}$ inhibition was determined in the hypothetical extreme case that $\mathrm{I}_{\mathrm{CaL}}$ inhibition was entirely due to (+)-thioridazine. Assuming no interaction between the enantiomers in the inhibition of $\mathrm{I}_{\mathrm{CaL}}$, a hypothetical dose-response curve of $\mathrm{I}_{\mathrm{CaL}}$ inhibition for (+)-thioridazine was created. This was identical to that determined for the racemate but shifted such that only half the concentration was required to reach the same inhibition. $\mathrm{I}_{\mathrm{Kr}}$ inhibition was then determined by optimization.

\section{Results}

Modeled drug effects on APD are shown in Figures 1 and 2. $\mathrm{I}_{\mathrm{Kr}}, \mathrm{I}_{\mathrm{Ks}}$, and $\mathrm{I}_{\mathrm{CaL}}$ inhibition are shown in Figure 3.

Using the forward method for the racemate, zero $\mathrm{I}_{\mathrm{CaL}}$ inhibition was determined. Given zero $\mathrm{I}_{\mathrm{CaL}}$ inhibition, a maximal level of $45 \% \mathrm{I}_{\mathrm{Kr}}$ inhibition was determined with higher dose. The accuracy of the fit was more accurate at low concentration than at higher concentrations. For (-)thioridazine, $10 \% \mathrm{I}_{\mathrm{CaL}}$ inhibition was determined and no $\mathrm{I}_{\mathrm{Kr}}$ inhibition was determined for any concentration. For $(+)$-thioridazine, $30 \% \mathrm{I}_{\mathrm{CaL}}$ inhibition was determined at $0.1 \mathrm{mg} \mathrm{L}^{-1}(\mathrm{RMSE}=3.2 \%)$. However, a level of $15 \% \mathrm{I}_{\mathrm{Kr}}$ inhibition was selected at this concentration, and an $\mathrm{I}_{\mathrm{CaL}}$ inhibition of $40 \%$ was determined given this assumption $(\mathrm{RMSE}=2.7 \%)$. The determined $\mathrm{I}_{\mathrm{Kr}}$ inhibition increased to a level of $85 \%$ with higher dose.

Inverse method A was used assuming equal $\mathrm{I}_{\mathrm{CaL}}$ inhibition between enantiomers. A maximal level of $30 \%$ $\mathrm{I}_{\mathrm{Kr}}$ inhibition was determined for (-)-thioridazine. For (+)thioridazine, no $\mathrm{I}_{\mathrm{Kr}}$ inhibition was determined at $0.1 \mathrm{mg} \mathrm{L}^{-}$ ${ }^{1}$ as the measured APD was shorter than that predicted by the $\mathrm{I}_{\mathrm{CaL}}$ inhibition. With increasing dose, $\mathrm{I}_{\mathrm{Kr}}$ inhibition of $80 \%$ was determined.

Inverse method B was used assuming the extreme case that $\mathrm{I}_{\mathrm{CaL}}$ inhibition was entirely attributable to (+)thioridazine. The dose-response curve was shifted to a half maximal inhibitory concentration of $0.12 \mathrm{mg} \mathrm{L}^{-1}$. $\mathrm{I}_{\mathrm{CaL}}$ inhibition at (+)-thioridazine concentrations of $0.1,1$, and $10 \mathrm{mg} \mathrm{L}^{-1}$ was calculated as $16.3 \%, 32.6 \%$, and $36.2 \%$. 


\section{Discussion}

For the racemate using the forward method, the determined $\mathrm{I}_{\mathrm{Kr}}$ inhibition was smaller than that reported in the literature, and the observed rate dependence of prolongation was not well described. Using the inverse method however, the model described the experimental data for the racemate with considerably greater accuracy. Simultaneous inhibition of $\mathrm{I}_{\mathrm{Kr}}$ and $\mathrm{I}_{\mathrm{CaL}}$ provided a better explanation for the observed pattern of rate dependence than $\mathrm{I}_{\mathrm{Kr}}$ inhibition alone. This supports the hypothesis that the measured changes in APD for the racemate were due to competing prolonging and shortening effects.

For (-)-thioridazine using the forward method, only a small degree of $\mathrm{I}_{\mathrm{CaL}}$ inhibition and no $\mathrm{I}_{\mathrm{Kr}}$ inhibition was determined. Using the inverse method, the $\mathrm{I}_{\mathrm{Kr}}$ inhibition was smaller than that determined for both the racemate and (+)-thioridazine. It was also smaller than the racemate induced $\mathrm{I}_{\mathrm{Kr}}$ and $\mathrm{I}_{\mathrm{Ks}}$ inhibition reported in the literature. Both methods resulted in an accurate fit to the data.

For (+)-thioridazine, reproducing the shortening at 0.1 $\mathrm{mg} \mathrm{L}^{-1}$ required more substantial inhibition of $\mathrm{I}_{\mathrm{CaL}}$. Using the forward method, the determined inhibition of $\mathrm{I}_{\mathrm{Kr}}$ was similar to the values of racemate induced $\mathrm{I}_{\mathrm{Kr}}$ inhibition obtained from the literature. The model could describe the experimental data only moderately well using this approach, as the reverse rate dependency of the shortening was greater than what could be accounted for by $\mathrm{I}_{\mathrm{CaL}}$ inhibition. Using the inverse method, the levels of $\mathrm{I}_{\mathrm{Kr}}$ inhibition determined at higher doses were very similar to those found using the forward method. However, the shortening observed at $0.1 \mathrm{mg} \mathrm{L}^{-1}$ meant that zero $\mathrm{I}_{\mathrm{Kr}}$ inhibition was determined. In addition, even attributing $\mathrm{I}_{\mathrm{CaL}}$ inhibition entirely to the (+)-enantiomer (inverse method B), the magnitude of the observed shortening could not be accurately accounted for.

Using the forward method, a greater degree of $\mathrm{I}_{\mathrm{Kr}}$ inhibition was determined for $(+)$-thioridazine than for the racemate. If the $\mathrm{I}_{\mathrm{Kr}}$ inhibition induced by the racemate is primarily due to (+)-thioridazine, a similar but left-shifted dose-response curve should be expected. The $\mathrm{I}_{\mathrm{Kr}}$ inhibition determined using the forward method was almost identical to that reported by Drolet et al. [4] for the racemate, as were the determined values at 1 and $10 \mathrm{mg}$ $\mathrm{L}^{-1}$ using the inverse method. Thus, the determined levels of $\mathrm{I}_{\mathrm{Kr}}$ inhibition for (+)-thioridazine were plausible.

Overall, the results show that the adapted model relatively accurately described the observed phenomena. The APD prolonging effects were well described, and simultaneous inhibition of $\mathrm{I}_{\mathrm{Kr}}$ and $\mathrm{I}_{\mathrm{CaL}}$ fit the data better than $\mathrm{I}_{\mathrm{Kr}}$ inhibition alone, even when no shortening was observed. However, the rate dependence of APD shortening for (+)-thioridazine at $0.1 \mathrm{mg} \mathrm{L}^{-1}$ dose was not well described. In addition, while there were differences between the forward and inverse methods, the determined levels of $\mathrm{I}_{\mathrm{Kr}}$ blockade agreed well between the methods and were plausible when compared to values obtained for the racemate from the literature. This indicates that the APD prolongation is well described by $\mathrm{I}_{\mathrm{Kr}}$ inhibition. The results therefore suggest that the experimental results may related to reduced $\mathrm{I}_{\mathrm{Kr}}$ inhibition due to (-)-thioridazine.

Thioridazine is effective in treatment of drug resistant tuberculosis [5], but cardiotoxic inhibition of the $\mathrm{I}_{\mathrm{Kr}}$ current is a concern. Considering that (-)-thioridazine was shown to have reduced anti-psychotic effect [6] and similar antimicrobial effects [7], investigation of the cardiotoxicity of the isolated enantiomers is needed.

\section{Conclusion}

Simultaneous inhibition of the $\mathrm{I}_{\mathrm{Kr}}$ and $\mathrm{I}_{\mathrm{CaL}}$ currents allowed for accurate replication of the observed effects of (-)-thioridazine and the racemate. Prolongation due to $(+)$-thioridazine was also well described by the model while the reverse rate dependence of shortening was not. Determined levels of $\mathrm{I}_{\mathrm{Kr}}$ inhibition were plausible when compared to data from the literature. The $\mathrm{I}_{\mathrm{Kr}}$ inhibition determined for (-)-thioridazine was substantially smaller than that determined for $(+)$-thioridazine or the racemate. These results suggest that the difference in prolongation may be related to difference in $\mathrm{I}_{\mathrm{Kr}}$ inhibiting effect.

\section{References}

[1] Jensen AS, Struijk JJ. Differential effects of thioridazine enantiomers on action potential duration in rabbit papillary muscle. Eur J Pharmacol 2015;747:7-12.

[2] Shannon TR, Wang F. A mathematical treatment of integrated ca dynamics within the ventricular myocyte. Pharm Res 2004;87(5):3351-71.

[3] Jensen AS, Struijk JJ. Adaptation of Rabbit Ventricular Cell Model to Reproduce Action Potentials in Isolated Papillary Muscle. Comput Cardiol 2015.

[4] Drolet B, Vincent F. Thioridazine lengthens repolarization of cardiac ventricular myocytes by blocking the delayed rectifier potassium current. J Pharmacol Exp Ther 1999;288(3):1261-8.

[5] Amaral L, Viveiros M. Why thioridazine in combination with antibiotics cures extensively drug-resistant mycobacterium tuberculosis infections. Int $\mathrm{J}$ Antimicrob Agents 2012;39(5):376-80.

[6] Thanacoody HKR. Thioridazine: The good and the bad. Recent Pat Antiinfect Drug Discov 2011;6(2):92-8.

[7] Kristiansen JE, Hendricks O. Reversal of resistance in microorganisms by help of non-antibiotics. J Antimicrob Chemother 2007;59(6):1271-9.

Address for correspondence.

Johannes Jan Struijk.

Fredrik Bajers Vej 7,

9220 Aalborg Ø, DK.

jjs@hst.aau.dk 